\title{
Adaptive optimisation of illumination beam profiles in fluorescence microscopy
}

\author{
T. J. Mitchell ${ }^{a}$, C. D. Saunter ${ }^{a}$, W. O’Nions ${ }^{a}$, J. M. Girkin ${ }^{a}$, G. D. Love ${ }^{a}$ \\ ${ }^{a}$ Centre for Advanced Instrumentation \& Biophysical Sciences Institute, Department of \\ Physics, Durham University, Durham DH1 3LE, UK
}

\begin{abstract}
Wide-field fluorescence microscope techniques such as single/selective plane illumination microscope (SPIM) are typically configured to image large regions of a sample at once. Here the illumination beam provides uniform excitation of several biological features across the region, 'sliced' to a thickness of between $5-10$ microns. In this paper we propose a simple alteration to the optical configuration of a SPIM by switching the light-sheetforming cylindrical lens with a spatial light modulator. This has the potential to adaptively reconfigure the light sheet geometry to improve the optical sectioning of specific biological features, rather than the thicker sectioning of several features at once across a larger observation field-of-view. We present a prototype version of such a system, referred to as an Adaptive-SPIM (A-SPIM) system. We then suggest that the direct recording of illumination beam shapes within the working microscope system can better facilitate the analysis and subsequent re-configuration of the illumination beam to a specific geometry, and summarise the design and operation of a device that we have developed specifically for this purpose. We finally present reconstructed quantitative three dimensional flux maps of illumination beams from three microscope configurations taken using this miniature high-dynamic range beam profiling device, comparing the beam geometry of a regular SPIM system with our prototype A-SPIM system, and suggesting future improvements.
\end{abstract}

Keywords: Fluorescence Microscopy, SPIM, Embryogenesis, Beam Shaping, Beam Diagnostics

\section{INTRODUCTION \& THEORY}

Optically-sectioning fluorescence microscopy (FM) techniques have given modern developmental biologists the ability to observe dynamic processes within living specimens at high spatial and temporal resolutions. ${ }^{1,2}$ Unprecedented lateral and axial spatial resolution are achieved with the cost of either a slow raster-scan based imaging procedure, as in the case of confocal or multi-photon point-scanning fluorescence microscopy, or an even slower computationally-intensive image reconstruction procedure, as in the case of super-resolution methods such as structured illumination microscopy. Conversely, in the study of time-sensitive phenomena such as the embryogenesis of model organisms, a high temporal resolution is preferred and the relatively modest lateral resolution is accepted as a reasonable expense for the improvement in imaging speed. One such technique that has become widely adopted due to its low phototoxicity and rapid imaging capability is single or selective plane illumination microscopy (SPIM). ${ }^{3}$

In SPIM, fluorescent excitation is provided by an illumination beam which is orthogonal to the axis of detection, as shown in Figure 1. A cylindrical lens is used to focus a collimated illumination laser beam in one lateral dimension, producing a sheet of light at the beam focus that illuminates a thin plane within the sample. ${ }^{4}$ Alignment of this excitation region within the focal depth of the orthogonal detection objective lens allows optical sections of the sample to be recorded; a three-dimensional (3D) image of the sample can be produced by scanning the sample through the beam. Although there have been a number of different incarnations of light sheet-based techniques, the most predominant advances concerning line-scanned illumination where the light sheet is built up across the detection field of view by scanning a circularly symmetric beam orthogonal to the propagation direction, SPIM remains the most rapid and simplest to implement of these techniques.

Further author information: (Send correspondence to T. J. Mitchell.)

T. J. Mitchell.: E-mail: t.j.mitchell@durham.ac.uk, Telephone: +44 (0) 1913343517

G. D. Love.: E-mail: g.d.love@durham.ac.uk, Telephone: +44 (0) 1913343696

Adaptive Optics and Wavefront Control for Biological Systems, edited by Thomas G. Bifano,

Joel Kubby, Sylvain Gigan, Proc. of SPIE Vol. 9335, 93350B · @ 2015 SPIE

CCC code: $1605-7422 / 15 / \$ 18 \cdot$ doi: $10.1117 / 12.2080310$

Proc. of SPIE Vol. 9335 93350B-1 
Light sheet dimensions:

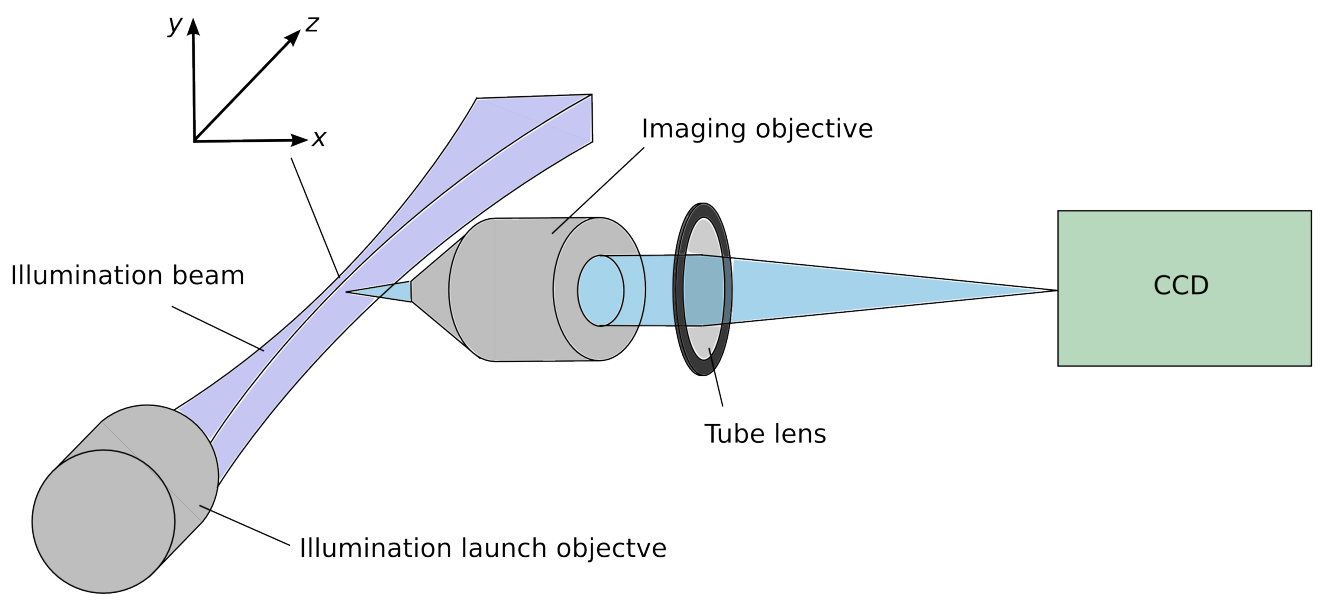

Figure 1. Illumination and detection geometry of a SPIM. A collimated illumination beam is focused in one dimension and relayed via an objective lens to the focal field of an orthogonal imaging system. The illumination beam shape can be parameterised according to its dimensions within the focal volume of the illumination objective lens: The sheet height is measured along y, the axis mutually orthogonal to both illumination and observation objective lenses; The sheet thickness is measured along $\mathrm{x}$; The sheet length is measured along $\mathrm{z}$, the optical axis of the illumination beam. The latter two dimensions of the light sheet are set by the beam waist and Rayleigh length respectively, and as such are coupled parameters.

Since Gaussian beam optics dictate that the Rayleigh range and waist of a focused Gaussian laser beam are inextricably linked, in a SPIM system this results in the propagation length of the light sheet within the sample being proportional to the optical slice thickness. Thus, in terms of the detected image, reducing the light sheet extent from a wide region of the sample down to a single cellular feature, results in an improvement in the optical sectioning of that particular feature as the sheet thickness reduces, and the increased numerical aperture (NA) of the illumination also help reduce shadowing artifacts in a similar, yet subtly different, fashion to multi-directional SPIM. ${ }^{5}$ Additionally, as biological specimens typically consist of many cellular surfaces and regions that have a distinct curvature, alteration of the light sheet focal length to locally match the position and topology of these features would result in feature-specific, well-sectioned images. However, to assess the success of such beam modifications, we need to directly quantify the beam shape within the confines of the microscope. We have previously developed a purpose built miniature camera probe, capable of reproducing a high dynamic range of flux values within such constrained volumes, demonstrating the operation of the device within a SPIM. ${ }^{6}$

In this paper we suggest an alternative SPIM setup where the light sheet illumination beam is formed by an adaptive optical element rather than a static cylindrical lens. The operation of our custom beam profiling device is summarised and we present additional results of using the probe to profile several microscope illumination configurations, using these results to discuss the preliminary configuration of our prototype Adaptive-SPIM system.

\section{PROPOSED ADAPTIVE-SPIM SYSTEM}

In order to produce an adaptively-shaped illumination light sheet, we constructed a prototype SPIM system where the cylindrical lens has been replaced by a spatial light modulator (SLM). As the beam's 'line-focus' is now produced by an adaptive optical element, the focal length can be altered across the height of the sheet. The macroscopic light sheet formed by the SLM (BNS, Colorado, USA) is then telescopically re-imaged by a 4-f relay to the scale appropriate for microscope sample illumination, as shown in Figure 2. The 4-f relay was comprised 


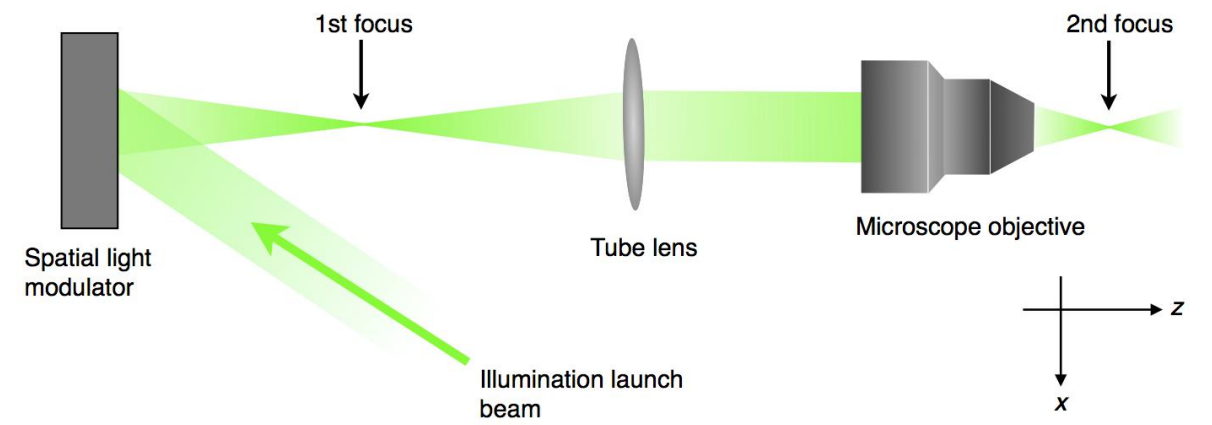

Figure 2. Schematic of the illumination beam optics in our prototype Adaptive-SPIM system. In this system a line focus is produced by focusing a collimated laser beam along one dimension only via a SLM. This line focus is then re-imaged via 4 -f relay to an appropriate microscopic scale.

of an achromatic doublet tube lens (focal length $250 \mathrm{~mm}$, Thorlabs, UK) and microscope objective lens (M:10x; NA:0.25; WD:10.5 mm; Nikon, Japan). Formation of the line focus directly from an adaptive element allows the focal length of the beam to be altered along the height of the light sheet.

\section{BEAM PROFILING DEVICE}

A detailed account of our beam profiling device and its operation is published elsewhere. ${ }^{6}$ In summary, we re-purposed a disposable sensor designed for endoscopy as a waterproof microscope beam probe and devised a quantitative high dynamic range (HDR) imaging procedure, calibrating the response of the sensor to a range of incident fluxes to allow the reconstruction of quantitative HDR flux maps. In short, our HDR imaging procedure is comprised of three main steps: First, we model the signal response of all sensor pixels to several uniform incident fluxes over a range of exposures. Next, we take images of the scene with a range of several exposures. Finally, for each pixel on the sensor we select the longest unsaturated exposure and use the known exposure time and recorded signal to numerically determine the best estimate of the flux incident at that pixel, via our sensor response model. By mounting the probe on an automated translation stage face-on to the optical axis of a beam, taking several exposures at any number of positions throughout the focal volume of the beam, a 3D beam profile can be recorded.

\section{PROFILING RESULTS \& DISCUSSION}

In this section the $z$-scans of different microscope beams are presented, rendered as 3D beam profiles with colourmaps corresponding to the reconstructed flux magnitude. All of these beam profiles were recorded using a range of 10 exposures at each of $60 z$-positions within the profiling volume: $0.18,0.27,0.45,0.73,1.09,1.82$, $3.27,6.18,11.82$, and $20.91 \mathrm{~ms}$. The reconstructed flux magnitude is a measure of the incident flux at the sensor relative to the fluxes used in the device calibration. ${ }^{6}$ In each figure the relative flux scale has been thresholded and scaled to make the viewing and comparison of the overall beam structures in each reconstruction easier: In Figs. $3 \& 4$ the maximum reconstructed fluxes are higher than the maximum presented colourbar value; in Fig.5 the maximum reconstructed flux at the beam focus matches the maximum presented colourbar value.

\subsection{System I: SPIM}

Fig. 3 shows beam profiles taken within the water tank of a dual-wavelength SPIM microscope. In this system two separate laser light sources were directed through the same illumination optics to provide illumination that could excite two types of fluorophore at once, or switch between the two. Thus, for the microscope performance to be matched at both wavelengths, the illumination beam profiles were required to possess the same dimensions. The illumination beam wavelength was $488 \mathrm{~nm}$ in the leftmost reconstruction and $532 \mathrm{~nm}$ in the rightmost reconstruction. Both beam profiles were taken over the same $1.0 \mathrm{~mm}$ axial range. 


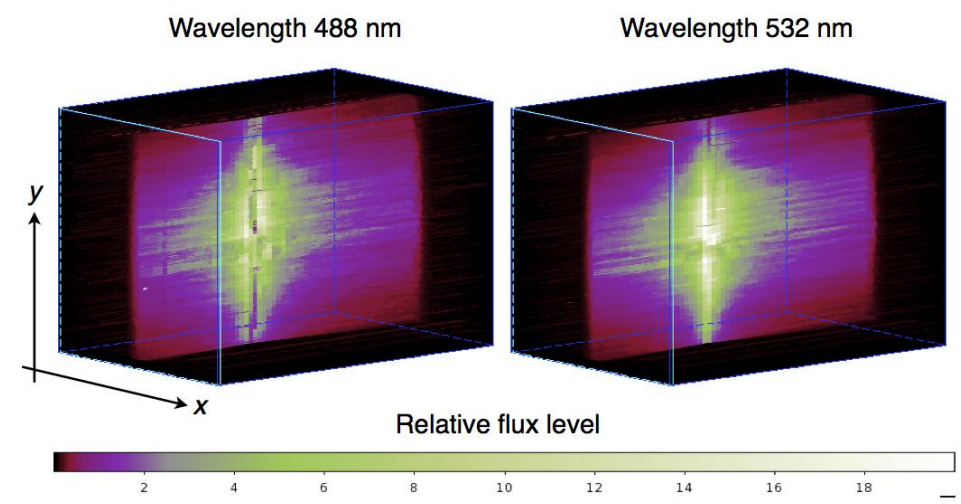

Figure 3. Reconstructed beam profiles taken within a dual-wavelength SPIM system. The colourbar represents the incident flux level, relative to the uniform fluxes used in the calibration of the sensor. The left profile was taken with the blue laser illumination (wavelength $488 \mathrm{~nm}$ ) and the right profile was taken with the green laser illumination (wavelength $532 \mathrm{~nm}$ ). The light sheets are well-aligned within the focal volume and have the same height, thickness, and length dimensions. The overall profiled volume has dimensions of $(x \times y \times z)$ are $0.74 \mathrm{~mm} \times 0.74 \mathrm{~mm} \times 1.0 \mathrm{~mm}$.

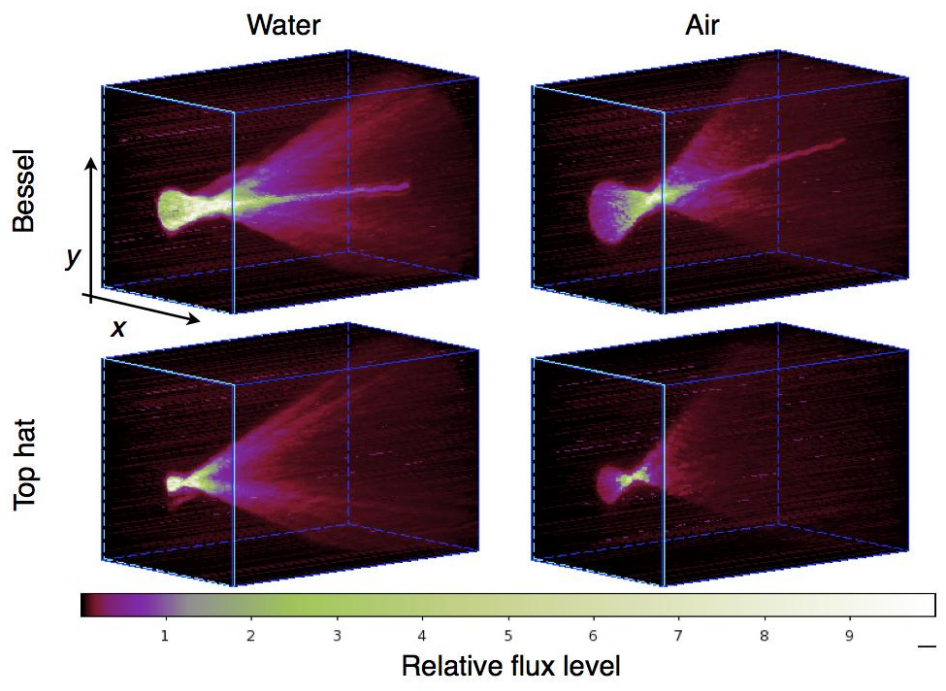

Figure 4. Reconstructed beam profiles taken within a microscope with switchable illumination beam geometry. The Bessel beams (top) feature a greatly extended bright focal region compared to the top hat beams (bottom). The axial extent of these beam profiles is $4.0 \mathrm{~mm}$. green (right, wavelength $532 \mathrm{~nm}$ ) light sheets are well-aligned within the focal volume and have the same height, thickness, and length dimensions. The overall profiled volume has dimensions of $(x \times y \times z)$ are $0.74 \mathrm{~mm} \times 0.74 \mathrm{~mm} \times 4.0 \mathrm{~mm}$.

The dual-wavelength illumination beams are clearly well-aligned within the system, producing a line focus at the same position within the focal volume that has the same sheet parameters: height, thickness, and length.

\subsection{System II: Bessel vs. top hat}

In Fig.4 the profiles of two illumination beam geometries are presented for comparison both with (leftmost profiles) and without (rightmost profiles) water immersion of the beam. In this microscope system a SLM was conjugated to the pupil of the illumination objective and switched between axicon and uniform phase patterns to alter the illumination between Bessel and top hat beams. A wavelength of $532 \mathrm{~nm}$ was used - the design wavelength of the chosen SLM. These beam profiles were taken over a $4.0 \mathrm{~mm}$ axial range. 


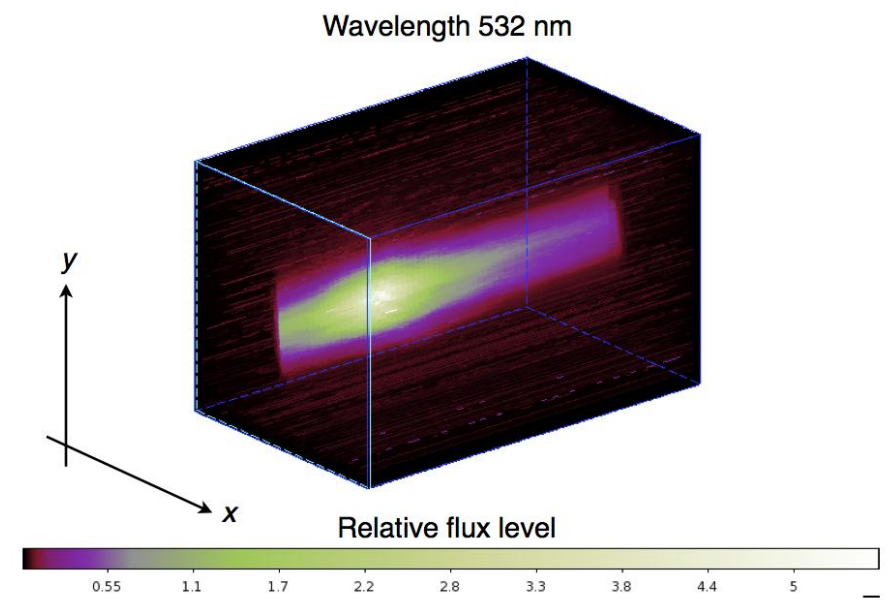

Figure 5. Reconstructed beam profile of the light sheet illumination formed using our prototype adaptive SPIM system. The overall profiled volume has dimensions of $(x \times y \times z)$ are $0.74 \mathrm{~mm} \times 0.74 \mathrm{~mm} \times 2.4 \mathrm{~mm}$.

The reconstructions clearly demonstrate the extended focal range of the Bessel beam illumination compared with that of the top hat illumination beam. Additionally, the profiles taken without water immersion demonstrate a distinct deviation of the beam direction and relative flux magnitude when compared with the water-immersed beam profiles.

\subsection{System III: Adaptive SPIM}

Fig.5 shows a reconstructed beam profile obtained from a preliminary configuration of our A-SPIM system set up to produce a simple line focus. This beam profile was taken over a $2.4 \mathrm{~mm}$ axial range. A comparison of the light sheet dimensions from our SPIM and prototype A-SPIM systems is given in Table 1.

Though the current beam produced in our prototype A-SPIM system does not match the dimensions of a regular SPIM beam geometry our results demonstrate the successful replacement of the cylindrical lens typically used in SPIM with an adaptive element. It is worth noting here that the $100 \mu \mathrm{m}$ light sheet height is actually well-suited to particular features of the zebrafish (Danio rerio), especially the eye lens. As such the illumination dimensions that need to be altered in future A-SPIM configurations for feature-specific optical sections are only the thickness and length of the light sheet. In the present configuration the rear aperture of the objective lens is under-filled, and thus the light sheet has a very low NA, resulting in a thick light sheet with a long length. Future configurations will reduce the thickness and length dimensions of the A-SPIM light sheet down past that of our regular SPIM beam by increasing the beam size across the rear aperture of the illumination objective, and using a higher NA objective lens if necessary, to improve the feature-specific optical-sectioning capabilities of the system. Following this we will implement adaptive alteration of the local focal position of the light sheet to match the curvature of specific features within biological specimens.

\section{SUMMARY}

We have suggested that adaptively-shaped illumination beams in fluorescence microscopy will allow improved sectioning of specific biological features within model organisms. For such an approach to be successful it is

Table 1. Comparison of illumination beam dimensions of light sheets formed in our SPIM and prototype Adaptive-SPIM system.

\begin{tabular}{l|ccc}
\hline System & Sheet thickness $(x) / \mu \mathrm{m}$ & Sheet height $(y) / \mu \mathrm{m}$ & Sheet length $(z) / \mu \mathrm{m}$ \\
\hline SPIM & 6 & 400 & 30 \\
\hline A-SPIM & 10 & 100 & 320 \\
\hline
\end{tabular}


desirable to directly obtain quantitative measurements of the 3D beam shape within the working microscope. We have presented further results of using a purpose-built beam probe within a range of microscope configurations, including a prototype Adaptive-SPIM system where the cylindrical lens of a SPIM was replaced by a SLM. Our Adaptive-SPIM system demonstrates the successful formation of a light sheet by an adaptive optical element; future configurations of the device will demonstrate specific alteration of the light sheet shape to match the curvature of individual cellular features within model organisms such as the zebrafish.

\section{ACKNOWLEDGMENTS}

This work was funded by the EPSRC, the British Heart Foundation Research Excellence Award (Edinburgh), and RCUK.

\section{REFERENCES}

[1] J. A. Conchello and J. W. Lichtman, "Optical sectioning microscopy.," Nat. Methods 2, pp. 920-931, 2005.

[2] B. R. Masters, "The Development of Fluorescence Microscopy," in eLS, pp. 1-9, J. Wiley \& Sons, Ltd, 2010.

[3] J. Huisken, J. Swoger, F. Del Bene, J. Wittbrodt, and E. H. K. Stelzer, "Optical sectioning deep inside live embryos by selective plane illumination microscopy.," Science 305(5686), pp. 1007-9, 2004.

[4] K. Greger, J. Swoger, and E. H. K. Stelzer, "Basic building units and properties of a fluorescence single plane illumination microscope," Rev. Sci. Instrum. 78(2), p. 023705, 2007.

[5] J. Huisken and D. Y. R. Stainier, "Even fluorescence excitation by multidirectional selective plane illumination microscopy (mSPIM)," Opt. Lett. 32(17), pp. 2608-2610, 2007.

[6] T. J. Mitchell, C. D. Saunter, W. O’Nions, J. M. Girkin, and G. D. Love, "Quantitative high dynamic range beam profiling for fluorescence microscopy.," Rev. Sci. Instrum. 85(10), p. 103713, 2014. 